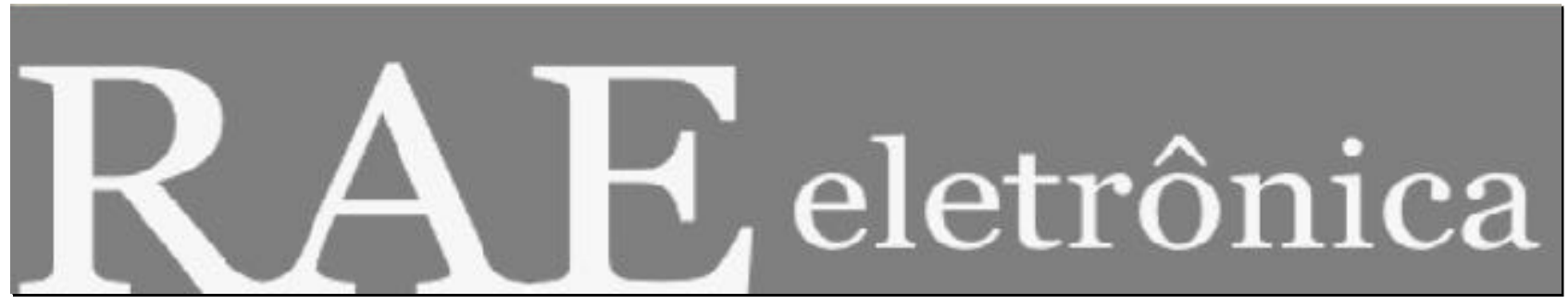

\title{
EVASÃO EM CURSO VIA INTERNET: EXPLORANDO VARIÁVEIS EXPLICATIVAS
}

Por:

\section{Gardênia Abbad, UNB}

Renata Silveira Carvalho, UNB

Thaís Zerbini, UNB

RAE-eletrônica, v. 5, n. 2, Art. 17, jul./dez. 2006

http://www.rae.com.br/eletronica/index.cfm?FuseAction=Artigo $\& I D=3652 \&$ Secao=ARTIGOS $\&$ Volu $\mathrm{me}=5 \&$ Numero $=2 \& \mathrm{Ano}=2006$

(C) Copyright, 2006, RAE-eletrônica. Todos os direitos, inclusive de tradução, são reservados. É permitido citar parte de artigos sem autorização prévia desde que seja identificada a fonte. A reprodução total de artigos é proibida. Os artigos só devem ser usados para uso pessoal e nãocomercial. Em caso de dúvida, consulte a redação: raeredacao@fgvsp.br.

A RAE-eletrônica é a revista on-line da FGV-EAESP, totalmente aberta e criada com o objetivo de agilizar a veiculação de trabalhos inéditos. Lançada em janeiro de 2002, com perfil acadêmico, é dedicada a professores, pesquisadores e estudantes. Para mais informações, consulte o site www.rae.com.br/eletronica.

RAE-eletrônica

ISSN 1676-5648

(C2006 Fundação Getulio Vargas - Escola de Administração de Empresas de São Paulo.

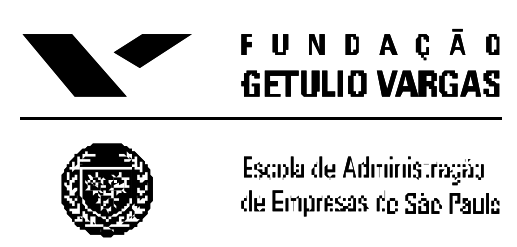




\title{
EVASÃO EM CURSO VIA INTERNET: EXPLORANDO VARIÁVEIS EXPLICATIVAS \\ Gardênia da Silva Abbad - Renata Silveira Carvalho - Thaís Zerbini
}

\section{RESUMO}

Apesar da importância dos cursos a distância como ferramenta de desenvolvimento de competências humanas para o trabalho, há poucas pesquisas que avaliam esses cursos e, em particular, os índices de evasão. O objetivo deste trabalho é identificar variáveis explicativas da evasão em um curso gratuito a distância, via Internet, ofe recido em nível nacional. Evasão refere-se à desistência definitiva do aluno em qualquer etapa do curso. As variáveis antecedentes incluíram dados demográficos e de uso dos recursos eletrônicos. Optourse pela análise de regressão logística pela natureza categórica da variável critério (concluinte versus não concluinte). Os resultados mostram que os alunos não concluintes são aqueles que tendem a não utilizar os recursos eletrônicos de interação (mural de notícias, chats, troca de mensagens eletrônicas). São discutidas as implicações teóricas e metodológicas dos achados, a necessidade de inclusão de variáveis motivacionais, cognitivas e contextuais, bem como suas possíveis interações em modelos multivariados explicativos de evasão.

PALAVRAS-CHAVE: Evasão, características individuais, recursos instrucionais via Internet, avaliação de treinamento a distância, treinamento via Internet.

\begin{abstract}
There are few studies on the evaluation of distance courses, especially dropout rates, in spite of their importance as a tool to develop human competences for the work. The purpose of the present paper is to identify variables that explain dropout rates in a free distance course offered through Internet at national level. In this study we consider dropout when the student quits definitely the course at any moment. The antecedent variables included demographic data and the use of electronic resources. We choose the analysis of logistic regression according to the categorical nature of the criterion variable (those who finished versus those who did not finish the course). The results show that students who did not finish the course are those who are not inclined to use the electronic resources of interactivity (notice boards, chats, exchange of electronic messages). The theoretical and methodological implications of the conclusions are discussed, as well as the need to include motivational, cognitive and contextual variables, and their possible interactions, in multivariate models explaining dropout.
\end{abstract}

KEYWORDS: Dropout, individual characteristics, learning resources using Internet, distance training evaluation, training using Internet. 


\section{EVASÃO EM CURSO VIA INTERNET: EXPLORANDO VARIÁVEIS EXPLICATIVAS \\ Gardênia da Silva Abbad - Renata Silveira Carvalho - Thaís Zerbini}

\section{INTRODUÇÃO}

A ampliação do acesso a treinamento, formação e qualificação profissionais vem sendo viabilizada pela aplicação das novas tecnologias de informação e comunicação (NTICs) em projetos de educação à distância. Os treinamentos mediados por computador (TBCs) e pela Web (TBWs), as ferramentas de gestão do conhecimento, somadas às tecnologias tradicionais de tornar disponível os conteúdos como o material impresso, materiais em vídeo e áudio, correspondência, vêm se tornando ferramentas imprescindíveis para capacitação e qualificação profissional da mão de obra.

Com as novas tecnologias de comunicação, como a Web e a Internet, rompem-se as barreiras de espaço e tempo e criam-se redes planetárias de comunicação entre pessoas residentes em diferentes partes do mundo. Os treinamentos podem utilizar essas novas ferramentas para aumentar as chances de que problemas e soluções sejam discutidos por gupos de pessoas temporal e espacialmente distantes entre si. Além disso, um treinamento mediado por novas tecnologias poderá estimular o indivíduo a navegar pela Internet para pesquisar em bibliotecas virtuais, acessíveis a todos aqueles que dispuserem de meios para viajar pelos caminhos desse ambiente cibernético.

O grande problema agora não é mais a localização e a disponibilização de informações relevantes, como ocorria até os idos de 1990, mas a sua transformação em conhecimento útil e aproveitável em curto espaço de tempo. Para isso, organizações disponibilizam meios de acesso às informações e conhecimentos relacionados à missão da empresa a todos os atores ligados a sua cadeia de valor, como fornecedores, parceiros, clientes, usuários ou beneficiários, além dos seus membros internos. Isso vem sendo feito com a criação de portais eletrônicos com acesso à Internet para todos ou quase todos os membros da organização e para a comunidade externa.

As ações de treinamento, desenvolvimento e educação (TD\&E) podem e devem apoiar-se nos sítios organizacionais para construir seus sistemas instrucionais modernos e flexíveis e nas redes de comunicação interna geradas pelos chats, fóruns, listas de discussão, recursos de apoio à resolução de problemas (FAQs - Frequently Asked Questions), videoconferências e redes internas de televisão. Abordagens construtivistas de treinamento podem ser colocadas em prática em função da multiplicidade de recursos eletrônicos que garantem conectividade e interação entre pessoas e equipes lotadas em unidades distantes, as quais podem produzir coletivamente novos conhecimentos e tecnologias de trabalho. Esse é um cenário propício à experimentação de novas formas de tornar disponíveis os conteúdos e estratégias de desenvolvimento de competências humanas para o trabalho. 


\section{EVASÃO EM CURSO VIA INTERNET: EXPLORANDO VARIÁVEIS EXPLICATIVAS \\ Gardênia da Silva Abbad - Renata Silveira Carvalho - Thaís Zerbini}

Recentemente, mais de cem universidades corporativas foram criadas no Brasil (Eboli, 2003). Além delas, também foram criadas universidades setoriais, voltadas para a educação de profissionais em áreas de atividades ou categorias profissionais específicas e para a promoção da gestão do conhecimento setorial.

As Instituições de Ensino Superior (IES), autorizadas pelo Ministério da Educação a disponibilizar aos alunos parte substantiva da carga horária de suas disciplinas na modalidade $a$ distância, iniciaram um processo de transposição de conteúdos para Universidades Virtuais. Apesar da importância do tema para as diversas áreas de pesquisa e atuação da Administração e áreas afins, há pequena produção de trabalhos sobre educação a distância, universidades corporativas e setoriais em congressos científicos em Administração. Algumas exceções a essa regra merecem destaque, como os trabalhos de Alperstedt e Plonski (2001), Maia e Meireles (2003) e Veiga et al. (1998).

As NTICs estão possibilitando a ampliação do acesso aos cursos por clientelas que antes, com a oferta de cursos presenciais, não eram alcançadas pelas ações educacionais ofertadas pelas organizações. Não é raro uma universidade corporativa disponibilizar seus cursos para milhares de colaboradores, pertencentes à sua cadeia de valor. Essa ampliação do acesso a formação, treinamento e educação para milhares de participantes simultaneamente gera, entretanto, grande responsabilidade para os profissionais de TD\&E nas organizações. Nesse contexto, um sucesso ou fracasso será percebido rapidamente por todos os participantes. Por isso, as avaliações formativas e aditivas sistemáticas são essenciais para que as ações educacionais, mediadas por novas tecnologias, não caiam em descrédito.

Porém, há pouco esforço dos pesquisadores e profissionais em avaliar as ações de TD\&E a distância, mediadas por novas tecnologias. Pouco se sabe, por exemplo, sobre as razões que levam os treinandos a abandonar os cursos antes de concluílos. Segundo Xenos et al. (2002) e Shin e Kim (1999), na Ásia os índices de evasão em cursos a distância chegam a atingir 50\%, enquanto na Europa está em torno de $20 \%$ a $30 \%$.

Esta pesquisa foi realizada com a finalidade de identificar variáveis explicativas de índices de evasão em um curso técnico a distância ofertado via Internet, em termos de características da clientela e de interação dos participantes com os recursos eletrônicos disponibilizados pelo curso. Para tal, foi aplicada uma análise de regressão lo gística visando identificar, entre algumas variáveis da clientela do curso, quais explicavam os índices de evasão. A seguir é apresentada uma breve análise da literatura sobre avaliação de treinamentos presenciais e a distância, que enfoca, ao final, assuntos ligados a retenção e evasão de participantes em cursos a distância. 


\section{EVASÃO EM CURSO VIA INTERNET: EXPLORANDO VARIÁVEIS EXPLICATIVAS \\ Gardênia da Silva Abbad - Renata Silveira Carvalho - Thaís Zerbini}

\section{BREVE ANÁLISE DA LITERATURA}

Em análises da literatura nacional e estrangeira, observa-se que, de modo geral, os sistemas de treinamento em ambientes organizacionais possuem as seguintes características: não utilizam análises sistemáticas de necessidades de treinamento, sem relacioná-las às competências críticas organizacionais ou as alinhá-las às estratégias organizacionais; fazem pequeno esforço de avaliação de aprendizagem e investem muito pouco em planejamento instrucional de cursos presenciais; utilizam estratégias e meios incompatíveis com o perfil da clientela e modelos de entrega presenciais, que privilegiam poucas pessoas lotadas em unidades diferentes da organização, em detrimento de modelos facilitadores da transferência horizontal e vertical de conhecimentos e novas aprendizagens.

Em decorrência dessa situação, cursos presenciais têm produzido resultados pouco animadores no nível de aprendizagem e impacto no desempenho do participante. Nos níveis de resultados e de valor final - conforme classificação de Hamblin (1978) -, há poucos estudos sobre avaliação de treinamentos presenciais ou a distância (Salas e Cannon-Bowers, 2001; Borges-Andrade, 2002; Freitas e Borges-Andrade, 2004; Mourão e Borges-Andrade, no prelo).

A avaliação da produção de conhecimentos sobre a eficácia de cursos a distância, em especial no que tange àqueles mediados pelas NTICs, mostrou poucos estudos sistemáticos sobre evasão, apesar de este ser um dos principais problemas e pontos de interrogação da área. Os poucos estudos existentes, entre os quais os trabalhos de Shin e Kim (1999), Xenos et al. (2002) e Zerbini e Abbad (2005), mostram que de modo geral há alta evasão em cursos a distância. Além disso, observam-se avaliações de aprendizagem imprecisas e inválidas; ausência de avaliação nos níveis de transferência ou impacto no desempenho, resultados e valor final; e pequena produção tecnológica em avaliação de TBWs e TBCs que, quando ocorre, se caracteriza pela criação de instrumentos de medida sem o necessário processo de validação psicométrica. Entre os estudos que relatam construção e aplicação de questionários estão alguns realizados em contexto de educação a distância como os de Warr e Bunce (1995), Cheung (1998, 2000), Dean e Webster (2000), Porras-Hernandez (2000) e outros em situações de treinamento em ambientes organizacionais, como Vargas (2000).

Quanto ao planejamento dos cursos, os estudos relatam o uso indevido de instrução e treinamento para fins de disseminação de informações e conhecimentos simples, que poderiam ser veiculados por portais eletrônicos ou por outros tipos de repositórios de conhecimentos; análise pouco 


\section{EVASÃO EM CURSO VIA INTERNET: EXPLORANDO VARIÁVEIS EXPLICATIVAS \\ Gardênia da Silva Abbad - Renata Silveira Carvalho - Thaís Zerbini}

sistemática da necessidade ou uso de procedimentos inadequados de mapeamento de competências como subsídio para o desenho das situações de aprendizagem; e aplicação indevida de treinamento em situações nas quais a incompetência do indivíduo não se deve à falta de habilidades ou motivação, mas a condições inadequadas de trabalho, como cultura, clima, suporte gerencial e material (para mais detalhes sobre esse tipo de resultados, veja Roullier e Goldstein, 1993, e Abbad et al., 2003).

A transposição dos conteúdos tradicionalmente expostos oralmente por professores ou instrutores em cursos presenciais para cursos mediados pela NTICs não tem sido uma tarefa fácil. Requer equipe treinada em desenho instrucional, especialistas em conteúdo, programadores visuais, profissionais da computação e ergonomistas, entre outros profissionais. Além disso, é necessário disponibilizar conteúdos, respeitando-se as características das mídias escolhidas.

Cursos de curta duração, com conteúdos pouco complexos, correm o risco de se transformar em folhetos informativos, com objetivos que não ultrapassam os níveis mais básicos das taxonomias de resultados de aprendizagem como conhecimento e compreensão (Bloom et al., 1972a, 1972b) ou informação verbal de Gagné (1985, 1988). Os conhecimentos disponibilizados pelos treinamentos, muitas vezes, não são desenhados de modo a possibilitarem acesso aos seus conteúdos após a realização do curso, não servindo, portanto, como job aids, mesmo quando os conteúdos são diretamente relacionados ao trabalho do participante. Nessas situações, as informações contidas no curso são de difícil recuperação pelo participante, que, em alguns casos, necessita fazer o curso inteiro para localizar uma única informação.

Além desses problemas específicos, relativos a cursos a distância baseados em NTICs, há aqueles herdados dos cursos presenciais, como exercícios incompatíveis com a natureza e grau de complexidade dos objetivos educacionais e a falta de informações sobre o perfil de entrada dos participantes quanto às suas características motivacionais, cognitivas, demográficas e profissionais, hábitos e estratégias de aprendizagem, resultando em um desenho de curso voltado para perfis genéricos, imprecisos e hipotéticos de clientela, entre outros problemas técnicos.

A baixa eficácia das ações educacionais a distância, mediadas por novas tecnologias, poderá levar essas ferramentas ao descrédito, tal como ocorreu anteriormente com a educação mediada pelo rádio e pela televisão. Entretanto, há pouco esforço dos pesquisadores e profissionais em produzir pesquisas na área de avaliação desse tipo de treinamentos (Sallas e Cannon-Bowers, 2001). A seguir são apresentadas as principais questões e resultados de pesquisas relativas à importância de características do treinamento e da clientela de cursos presenciais e a distância na explicação de resultados de treinamentos e evasão. 


\section{EVASÃO EM CURSO VIA INTERNET: EXPLORANDO VARIÁVEIS EXPLICATIVAS \\ Gardênia da Silva Abbad - Renata Silveira Carvalho - Thaís Zerbini}

\section{Características do treinamento}

São dois os tipos de trabalhos característicos dessa vertente de pesquisas. O primeiro se caracteriza pela produção de tecnologias relacionadas ao planejamento instrucional como estabelecimento de objetivos, escolha de estratégias de ensino, desenvolvimento e escolha de métodos e técnicas de treinamento e análise de relações de custo-benefício dos recursos investidos no treinamento. O segundo se caracteriza pela preocupação em estudar os processos psicológicos básicos subjacentes à aprendizagem e à transferência de treinamento. Entre os principais resultados do primeiro tipo de pesquisa - principal foco deste artigo - estão aqueles que demonstram o poder explicativo de certos aspectos do treinamento nos níveis de aprendizagem e transferência de treinamento.

De acordo com Abbad et al. (2003), os seguintes procedimentos ou estratégias instrucionais mantêm correlação positiva, estatisticamente significativa com transferência de aprendizagem: exemplificação; sumarização e/ou apresentação de organizadores gráficos, mapas e diagramas de apoio à memorização; diversificação de problemas; distinção entre problemas em que as habilidades ensinadas devem ser aplicadas e onde não são aplicáveis; apresentação das etapas do processamento de informações (codificação, inferência, mapeamento e aplicação); ensino de estratégias de autogerenciamento e estabelecimento de objetivos; explicações teóricas (indução de esquema geral) e apresentação de feedback (indução de esquema específico); e aplicação de diversas combinações de explicação $e$ feedback e estabelecimento de objetivos de ensino.

Em relação a métodos de treinamento, Cauble e Thurston (2000) estudaram o efeito de um treinamento interativo via computador sobre atitudes, aquisição de conhecimentos e eficácia instrucional. Os autores encontraram que o uso dessa tecnologia facilitou a aquisição dos conhecimentos e o desenvolvimento de um alto senso de competência entre os participantes do curso.

No Brasil há poucos trabalhos sobre características do treinamento, entre os quais pode ser citado o de Pantoja et al. (2001). Os resultados mostraram que a característica do treinamento formulação de objetivos e número de avaliações - foi um dos componentes que explicou uma porção pequena, mas significativa, da variabilidade de impacto do treinamento no trabalho. Também são poucos os relatos de pesquisa sobre cursos a distância, merecendo destaque para Vargas (2000), que descreve a implantação e avaliação de um curso a distância por videoconferência. Os resultados indicaram grande satisfação dos alunos quanto à modalidade de ensino, contudo mais da metade dos participantes afirmou que teria participado do curso mais ativamente caso este fosse presencial. Em 


\section{EVASÃO EM CURSO VIA INTERNET: EXPLORANDO VARIÁVEIS EXPLICATIVAS \\ Gardênia da Silva Abbad - Renata Silveira Carvalho - Thaís Zerbini}

relação ao desenvolvimento do curso (profundidade das discussões, equipamentos, qualidade, atendimento), ao local do curso (conforto e limpeza) e aos participantes (envolvimento e intenção), todos os itens foram bem avaliados.

Há modelos de desenho instrucional que sugerem critérios de avaliação específicos para projetos instrucionais na Web. Hall et al. (2003) sugerem um desses modelos, denominado Modelo de Desenho da Aprendizagem na Web, que se fundamenta em vários critérios de avaliação de desenhos instruciona is. Um desses critérios é a simplicidade, que engloba os conceitos de: (1) usabilidade (refere-se aos fatores do desenho do programa na interface gráfica do ambiente Web que tornam a tarefa do aprendiz mais simples e livre de estresse); e (2) consistência (refere-se à simplicidade com que os elementos de diferentes níveis de informação estão organizados no site que hospeda o curso, de modo que o uso de hipermídia - hipertexto e múltiplas mídias - facilite a tarefa de realização do curso e das atividades previstas na programação).

Porém, ainda para Hall et al. (2003), o desenho na Web deve se equilibrar entre duas polaridades: a simplicidade e a complexidade. Assim, um desenho instrucional deveria garantir ao aprendiz um acesso progressivo a aspectos mais complexos dos conteúdos. Nesse modelo, a complexidade é uma variável composta por três categorias, a saber: (1) a interatividade, que se refere ao quanto o desenho evita colocar o aprendiz em uma posição passiva de escuta ou leitura, mas possibilita a sua participação ativa nas atividades do curso, por exemplo, estimulando-o a realizar pesquisas na Web por meio de estruturas hipertextuais; (2) a multimodalidade, que se refere à apresentação dos conteúdos por intermédio de múltiplas modalidades e formatos (áudio, vídeo e texto), o que aumentaria a motivação para aprender, a aprendizagem e a retenção de conteúdos complexos; e (3) a adaptabilidade, que avalia o quanto o desenho possibilita ajustes às características dos diferentes perfis de clientelas e estilos de aprendizagem. Além disso, um desenho instrucional em ambiente da Web deveria estimular a autonomia do aprendiz e a interação tutor-aluno e aluno-aluno. Os resultados desse tipo de modelo de desenho instrucional ainda não são apoiados por pesquisas empíricas, porém têm sido utilizados como parâmetro para criação de objetos de aprendizagem na Web.

Scapin e Bastien (1993), preocupados em avaliar a qualidade ergonômica de interfaces homemcomputador, sugeriram oito critérios de navegabilidade e usabilidade desses sistemas, alguns dos quais foram utilizados por Carvalho e Abbad (2006) para avaliar as reações dos participantes à interface gráfica de um TBW. Nomeadamente os critérios são condução, carga de trabalho, controle explícito, adaptabilidade, gestão de erros, homogeneidade/coerência, significado dos códigos e denominações e compatibilidade. A variável de reação à interface gráfica foi testada em um modelo de regressão 


\section{EVASÃO EM CURSO VIA INTERNET: EXPLORANDO VARIÁVEIS EXPLICATIVAS \\ Gardênia da Silva Abbad - Renata Silveira Carvalho - Thaís Zerbini}

explicativo dos resultados do curso avaliado, porém não foi encontrada nenhuma influência significativa dessa variável na explicação de resultados de treinamento. Tal resultado foi explicado em parte pela natureza do curso avaliado, necessitando, portanto, de mais pesquisas utilizando a mesma medida, para verificar o poder preditivo da interface gráfica.

Em suma, as características do desenho instrucional afetam resultados de treinamento, porém o fazem com pequeno poder explicativo. Pouco se sabe sobre a influência exercida pelo uso das NTICs nos níveis de eficácia de cursos a distância. Há resultados não conclusivos, indicando, entretanto, que treinamentos desse tipo não são melhores nem piores que os presenciais em termos dos resultados de aprendizagem que são capazes de gerar. No entanto, há uma diferença entre as modalidades de cursos com presença e a distância - que merece destaque especial: os índices de evasão dos cursos. Em cursos a distância, esses índices são bem maiores que os encontrados em cursos presenciais.

\section{Características da clientela}

Abbad et al. (2003), em revisão de literatura sobre avaliação de treinamento, apresentam as principais variáveis explicativas de resultados de treinamento nas diversas linhas de pesquisa sobre TD\&E. Com relação às características da clientela, podem ser citadas: as características demográficas e profissionais, os repertórios de entrada, o interesse em aplicar o aprendido, a satisfação com o trabalho que executa dentro da organização que oferta os treinamentos, a auto-eficácia, o lócus de controle e a motivação para o treinamento. A investigação dessa linha de pesquisa cresceu bastante nos últimos anos. No Brasil, geralmente, as contribuições dessas variáveis na explicação de resultados de treinamento têm sido pequenas se comparadas às contribuições de variáveis ambientais, como suporte psicossocial à transferência de treinamento.

É prática comum entre os pesquisadores da área a coleta de dados demográficos para avaliar treinamentos. No entanto, essas variáveis têm apresentado efeitos estatisticamente controlados e não entram como variáveis explicativas em modelos estatísticos multivariados de investigação. Além disso, existem poucos estudos, tanto nacionais como estrangeiros, que relacionem tais dados com resultados de treinamento. Entre os estudos que investigam características demográficas dos participantes de cursos a distância estão Shea et al. (2001) e Xenos et al. (2002).

Warr e Bunce (1995) relataram um estudo feito em sistema de aprendizagem aberta, em que a idade está negativamente correlacionada com a aprendizagem. Resultados similares a estes foram 


\section{EVASÃO EM CURSO VIA INTERNET: EXPLORANDO VARIÁVEIS EXPLICATIVAS \\ Gardênia da Silva Abbad - Renata Silveira Carvalho - Thaís Zerbini}

encontrados em estudos brasileiros de avaliação de treinamentos presenciais (Abbad, 1999; Pantoja et al., 2001).

Entre as variáveis da clientela, as motivacionais são as que têm mostrado maior valor explicativo de aprendizagem e impacto de treinamentos, como afirmam Salas e Cannon-Bowers (2001). Lacerda e Abbad (2003) encontraram resultados em sua pesquisa que destacam a importância da motivação para aprender e do valor instrumental do treinamento para a aplicação de conhecimentos adquiridos em programas de treinamentos presenciais. Motivação para o treinamento também teve contribuição na explicação dos níveis de avaliação individual do treinamento em diversos estudos, entre eles Abbad et al. (2001), Sallorenzo (2000) e Warr e Bunce (1995).

A disposição dos indivíduos também parece interferir nos resultados do treinamento, como identificaram Colquitt et al. (2000). A conscienciosidade (fator de personalidade) e a ansiedade exercem efeito indireto sobre a motivação para aprender e os resultados do treinamento. Esses autores encontraram relações entre idade e motivação para aprender: alunos mais velhos demonstraram menor motivação, aprendizagem e eficácia pós-treinamento. Quanto à idade, Salas e Cannon-Bowers (2001) fazem uma ressalva, afirmando que os procedimentos do treinamento podem estar interferindo no modo como pessoas mais velhas aprendem.

Foram encontrados poucos resultados de pesquisas científicas sobre a participação de características individuais em treinamentos a distância, entre os quais os trabalhos de Xenos et al. (2002), Shin e Kim (1999) e Bernarth e Rubin (2004). Entre os resultados de Xenos et al., idade, gênero, conhecimento anterior relacionado ao conteúdo do curso, uso de recursos da informática para estudar e capacidade de administrar o próprio tempo dedicado ao estudo apareceram como os mais salientes. O estudo refere-se a níveis de evasão de alunos em um curso de graduação em informática, ofertado por uma universidade aberta grega. A direção desses relacionamentos será discutida mais adiante.

A pesquisa de Shin e Kim (1999), realizada com uma amostra de estudantes da Universidade Nacional Aberta da Coréia, mostrou que o nível de participação do aluno em atividades presenciais face a face estava associado ao nível de abandono do curso, de modo que os não concluintes do curso tenderam a ser aqueles que participaram menos desse tipo de atividade. $\mathrm{Na}$ seção seguinte, estão descritos alguns estudos sobre evasão de participantes de cursos a distância.

\section{Evasão em cursos a distância}




\section{EVASÃO EM CURSO VIA INTERNET: EXPLORANDO VARIÁVEIS EXPLICATIVAS \\ Gardênia da Silva Abbad - Renata Silveira Carvalho - Thaís Zerbini}

As pesquisas sobre evasão em cursos à distância localizadas na literatura especializada têm sido realizadas por pesquisadores da área de educação. A seguir, são descritas duas pesquisas cujos resultados serão comparados aos obtidos neste artigo.

Para Xenos et al. (2002), a evasão (dropout), principal preocupação de instituições de ensino a distância, é causada por múltiplos fatores endógenos e exógenos ao curso. Há alguns resultados relevantes de pesquisas, citados por esses autores, indicando que um dos principais fatores que afetam a evasão é a quantidade de módulos completada pelo aluno. As pesquisas mostram, por exemplo, que, na maior parte dos casos, os estudantes que interromperam sua participação em um curso a distância o fizeram logo após o prime iro ou segundo módulo.

Há fatores que, historicamente, vêm afetando os níveis de evasão em cursos universitários a distância e que podem ser classificados em três grandes categorias, conforme Xenos et al. (2002): (1) fatores internos relacionados às percepções do aluno e seu lócus de controle - interno-externo; (2) fatores relativos ao curso e aos tutores; e (3) fatores relacionados a certas características demográficas dos estudantes, como idade, sexo, estado civil, número de filhos, tipo de trabalho ou profissão, entre outras. Os fatores mencionados em 1 e 3 correspondem a características da clientela, enquanto os fatores definidos em 2 se referem às características do treinamento.

A pesquisa bibliográfica de Xenos et al. (2002) mostrou que, entre os fatores internos citados por pesquisadores como explicativos de evasão, estão: a percepção de dificuldade do curso, a motivação, a persistência do aluno e seu lócus de controle. Resultados interessantes, porém não conclusivos, mostram que as mulheres tendem a persistir mais do que os homens nos cursos. Quanto às características do desenho instrucional, os autores observaram que os níveis de evasão em cursos a distância são influenciados por fatores ligados ao desempenho do tutor. Aqui são mencionadas qualid ade e quantidade de apoio oferecido ao estudante, além do meio e tipo de contato utilizado pelo tutor em suas interações com os alunos. Há ainda os fatores internos ao curso, ligados aos seus procedimentos, como carga de trabalho, quantidade e dificuldade dos trabalhos escritos exigidos.

O estudo empírico de Xenos et al. (2002) - realizado em uma amostra de estudantes de um curso de graduação em Informática - avaliou o rendimento e a evasão de alunos. Evasão, nesse artigo, foi definida como o percentual de alunos que se matricularam no curso, mas nunca o iniciaram, ou que o começaram, mas interromperam sua participação. O curso avaliado teve um índice de 7,9\% de alunos na primeira categoria e de 20,5\% na segunda. Os principais resultados mostram que maiores índices de evasão ocorreram entre os alunos mais idosos, com idade igual ou superior a 35 anos; entre os homens, que mostraram maiores índices de evasão que as mulheres; entre os alunos que estudaram menos 


\section{EVASÃO EM CURSO VIA INTERNET: EXPLORANDO VARIÁVEIS EXPLICATIVAS \\ Gardênia da Silva Abbad - Renata Silveira Carvalho - Thaís Zerbini}

freqüentemente por meio do computador; entre os alunos que trocaram menos e-mails com os tutores e demais participantes do curso; e entre aqueles que tiveram pouco contato com atividades educativas na área de informática antes do curso. O estado civil não influenciou os níveis de evasão.

Entre os fatores externos, citados como razões da evasão pelos alunos em entrevistas individuais, havia os problemas profissionais, como troca de emprego e pressões de tempo; pessoais, como nascimento de filhos e morte na família; e problemas de saúde. Entre os fatores internos ao curso, a evasão deveu-se à falta de assistência do tutor e à quantidade de trabalhos escritos exigidos pelo curso. Os resultados mostraram que os evadidos possuem um perfil identificável no início do curso. Novos estudos são necessários para verificação da consistência desses achados em outros contextos. O presente trabalho é parte desse esforço de pesquisa. A pesquisa foi realizada com o intuito de verificar a influência de algumas características da clientela e do curso sobre a evasão em um curso a distância.

Outro estudo que merece ser mencionado é o de Shin e Kim (1999). Ao realizarem a avaliação de um curso de graduação na Universidade Nacional Aberta da Coréia, os autores definem as variáveis que influenciam evasão em cursos a distância como variáveis exógenas e endógenas. Em sua pesquisa, avaliaram três tipos de variáveis exógenas: (1) carga de trabalho, definida como a percepção do participante sobre o grau de exigência do trabalho que executa em seu emprego; (2) integração social, medid a em termos das percepções que o participante possui sobre o apoio e encorajamento que recebe das pessoas que o rodeiam para estudar e sentir-se parte da universidade; e (3) anseio, compreendida como o desejo do aluno de concluir o curso.

As variáveis endógenas foram definidas como tempo de estudo, entendido como a quantidade e o padrão de administração do tempo de estudo que o aluno adotou durante o semestre; planejamento da aprendizagem, que se refere ao grau de organização dos projetos individuais de aprendizagem, elaborados pelos estudantes; e atividades face a face, que incluem a avaliação do quanto os alunos participaram de palestras complementares e o quanto necessitaram buscar apoio de outros colegas e escolas locais.

Shin e Kim (1999) testaram um modelo de análise de trajetória (path analysis) para explicar rendimento de alunos da universidade coreana mencionada anteriormente, e uma análise de regressão logística para investigar os fatores associados à evasão de alunos. Das sete variáveis antecedentes carga de trabalho, integração social, anseio, tempo de estudo, planejamento da aprendizagem, atividades face a face e nota ou rendimento acadêmico -, apenas a participação do aluno em atividades face a face esteve associada negativamente com a evasão. Integração social esteve associada à evasão apenas ao final do primeiro semestre avaliado na pesquisa. Os autores discutem esses resultados 


\section{EVASÃO EM CURSO VIA INTERNET: EXPLORANDO VARIÁVEIS EXPLICATIVAS \\ Gardênia da Silva Abbad - Renata Silveira Carvalho - Thaís Zerbini}

destacando o importante papel de atividades presenciais na manutenção de alunos em cursos a distância.

A análise dessa amostra de artigos sobre a avaliação de treinamentos a distância revela pequena produção científica e tecnológica na área. Há poucas pesquisas que identificaram variáveis explicativas dos efeitos de treinamentos a distância. Muitos instrumentos de avaliação, utilizados nessas pesquisas, não foram submetidos à validação empírica. Foram localizados poucos trabalhos acerca das razões associadas aos altos índices de evasão de alunos nesses cursos. A análise da literatura indica avanços significativos apenas na produção de conhecimentos sobre treinamentos presenciais.

\section{PROCEDIMENTOS METODOLÓGICOS}

A pesquisa é de natureza exploratória e busca correlações e de campo, tendo sido realizada no período de janeiro a dezembro de 2002. Consistiu na avaliação de um curso gratuito, realizado totalmente a distância, via Internet, com tutoria ativa e que visava a ensinar a clientela a elaborar um plano de negócios. A duração prevista para realização do curso foi de 40 horas, distribuídas em 60 dias. Constavam cinco módulos seqüenciais, cada um composto por dois a cinco capítulos, acessíveis ao aluno no portal eletrônico do curso e/ou por meio de download do material. Além dos materiais e exercícios, o portal continha os seguintes recursos eletrônicos de interação (aluno-aluno, aluno-tutor, aluno-tutor-material): tutoria ativa, lista de discussão, mural de notícias, chat e troca de mensagens.

O modelo de investigação proposto neste estudo é composto por três componentes: (1) Características Demográficas da Clientela; (2) Uso dos Recursos Eletrônicos do Curso; e (3) Evasão (veja a Figura 1).

Figura 1 - Modelo proposto de investigação de variáveis explicativas de Evasão.

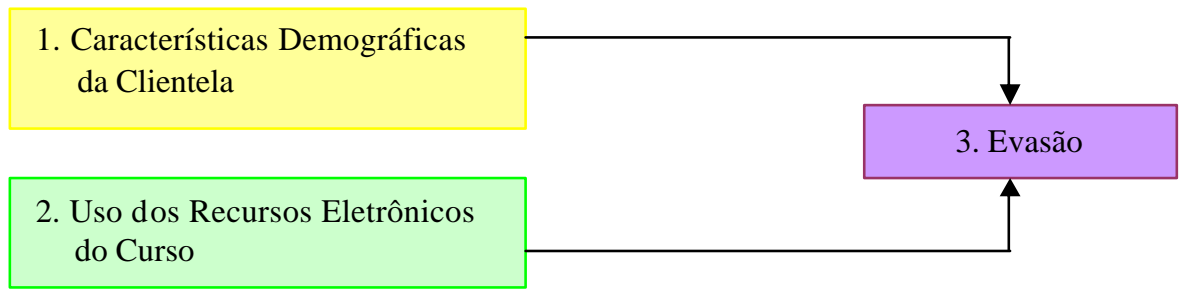




\section{EVASÃO EM CURSO VIA INTERNET: EXPLORANDO VARIÁVEIS EXPLICATIVAS \\ Gardênia da Silva Abbad - Renata Silveira Carvalho - Thaís Zerbini}

Características da Clientela refere-se aos dados demográficos dos participantes, como sexo, idade, região geográfica e nível de escolaridade. O Uso dos Recursos Eletrônicos do Curso diz respeito à frequiência de utilização espontânea das ferramentas: chats, listas de discussão e ambiente eletrônico do curso (website e webpages) durante todo o período do curso. Essas variáveis são medidas em termos do número de mensagens enviadas pelo aluno para a lista de discussão, do número de participações nos chats e do número de consultas espontâneas do aluno ao ambiente eletrônico do curso. O terceiro componente, Evasão, variável critério neste estudo, refere-se à desistência definitiva do aluno em qualquer etapa do curso, medida em termos da quantidade de alunos concluintes e não concluintes do curso.

As análises foram realizadas com a população de alunos inscritos no curso $(n=19.849)$. A Tabela 1 apresenta o número de concluintes e não concluintes do curso e as características do universo estudado.

Tabela 1 - Resultados descritivos da população $(n=19.849)$.

\begin{tabular}{|c|c|c|c|c|c|}
\hline Concluinte & $\mathrm{F}^{*}$ & $\%$ & Idade & & \\
\hline Sim & 11.597 & 51,9 & Média & 33,05 & \\
\hline Não & 9.996 & 44,7 & Desvio Padrão & 9,29 & \\
\hline Omisso & 766 & 3,4 & Omisso & 1.687 & \\
\hline Sexo & & & Lista de Discussão & $\mathrm{F}$ & $\%$ \\
\hline Masculino & 14.398 & 64,4 & 0 & 11.325 & 50,7 \\
\hline Feminino & 7.194 & 32,2 & Acima de 0 & 10.266 & 45,9 \\
\hline Omisso & 767 & 3,4 & Omisso & 768 & 3,4 \\
\hline Região Geográfica & & & Chat & & \\
\hline Sudeste & 11.864 & 53,1 & 0 & 13.285 & 59,4 \\
\hline Outras & 9.683 & 43,3 & Acima de 0 & 7.707 & 34,5 \\
\hline Omisso & 812 & 3,6 & Omisso & 1.367 & 6,1 \\
\hline Nível de Instrução & & & Ambiente do Curso & & \\
\hline Até $2^{\circ}$ grau & 5.129 & 22,9 & 0 a 10 & 8.375 & 37,5 \\
\hline $3^{\circ}$ grau incompleto & 7.527 & 33,7 & 11 a 20 & 6.065 & 27,1 \\
\hline $3^{\circ}$ grau e pós & 9.344 & 41,8 & Acima de 20 & 7.152 & 32,0 \\
\hline Omisso & 359 & 1,6 & Omisso & 767 & 3,4 \\
\hline \multicolumn{6}{|l|}{ Mural de Notícias } \\
\hline 0 a 10 & 10.435 & 46,7 & & & \\
\hline Acima de 10 & 10.757 & 48,1 & & & \\
\hline Omisso & 1.167 & 5,2 & & & \\
\hline
\end{tabular}

Observa-se que 44,7\% da população de inscritos no curso não concluíram o curso, o que indica um alto índice de evasão. A maior parte da amostra, que concluiu o curso (51,9\%), é do sexo masculino $(64,4 \%)$, residente na Região Sudeste $(53,1 \%)$, e possui média de idade de 33 anos (DP =9,29). Grande 


\section{EVASÃO EM CURSO VIA INTERNET: EXPLORANDO VARIÁVEIS EXPLICATIVAS \\ Gardênia da Silva Abbad - Renata Silveira Carvalho - Thaís Zerbini}

parte da população possui como último nível de instrução o $3^{\circ}$ grau completo ou pós-graduação $(41,8 \%)$. Quanto à participação nos recursos eletrônicos do curso, grande parte da população acessou mais de 10 vezes o mural de notícias $(46,7 \%)$ e de 0 a 10 vezes o ambiente eletrônico do curso $(37,5 \%)$. A maioria dos participantes não enviou mensagens para lista de discussão $(50,7 \%)$ e não participou dos chats $(59,4 \%)$.

De modo a avaliar o efeito do tamanho elevado da população $(\mathrm{N}=19.849)$ sobre a significância das correlações entre variáveis e a consistência dos resultados (erro tipo I), Tabachnick e Fidell (2001) sugerem que se realizem as mesmas análises com amostras aleatórias extraídas da população estudada. Sendo assim, nesta pesquisa foram repetidas as análises de regressão logística em duas amostras extraídas da população (amostra 1: $\mathrm{n}=1.349$; amostra 2: $\mathrm{n}=1.436$ ). As amostras são aleatórias simples e foram geradas pelo próprio programa de análise SPSS for Windows, versão 10.0.

Os dados utilizados neste estudo são de origem secundária, extraídos do gerenciador eletrônico do curso. Essas informações, transformadas em códigos numéricos, foram utilizadas como variáveis do modelo de investigação apresentado na Figura 1. Os dados referentes a essas variáveis foram transpostos para um arquivo eletrônico do programa $S P S S$, o que possibilitou a realização das análises estatísticas.

Foram realizadas análises descritivas e exploratórias para investigar a exatidão da entrada dos dados, a presença de casos extremos, a distribuição dos casos omissos e as características de distribuição de freqüência das variáveis. Seguindo orientação de Tabachnick e Fidell (2001), optou-se pela análise de regressão logística em função da natureza categórica da variável critério (concluinte versus não concluinte).

O primeiro conjunto de variáveis antecedentes corresponde a variáveis demográficas da clientela do curso. São elas: sexo, idade, nível de instrução e região geográfica de residência. A variável do sexo é definida como (1) masculino e (2) feminino. A idade é variáve l contínua e o nível de instrução foi coletado como variável categórica com 7 (sete) opções de resposta variando de " $1^{\circ}$ grau incompleto" a "pós-graduação". Após a realização de análises descritivas, percebeurse que essa variável apresentava poucos casos em algumas categorias, o que inviabilizaria a aplicação da análise de regressão logística. Assim, a variável nível de instrução foi recodificada em três categorias, de modo a garantir uma distribuição mais equilibrada. São elas: (1) de $1^{\circ}$ grau incompleto a $2^{\circ}$ grau completo; (2) $3^{\circ}$ grau incompleto; e (3) $3^{\circ}$ grau completo e pós-graduação. O mesmo procedimento foi seguido para a variável região geográfica de residência, transformando-a em variável dicotômica (1 - Região Sudeste; 2 - demais regiões). 


\section{EVASÃO EM CURSO VIA INTERNET: EXPLORANDO VARIÁVEIS EXPLICATIVAS \\ Gardênia da Silva Abbad - Renata Silveira Carvalho - Thaís Zerbini}

O segundo conjunto de variáveis antecedentes do modelo se refere à freqüência com que os participantes utilizaram os recursos eletrônicos do curso. Todas as variáveis desse conjunto tiveram seus valores classificados em categorias com intervalos maiores, garantindo um mínimo de casos em cada categoria, o que é exigido pela análise de regressão logística. Não foi possível investigar o poder explicativo do contato do aluno com o tutor, uma vez que $90 \%$ dos participantes não utilizaram espontaneamente esse recurso durante o treinamento.

As oito variáveis pertencentes aos dois conjuntos anteriormente descritos entraram na análise de regressão logística como variáveis antecedentes ou explicativas de evasão ( 1 - concluintes e 2 - nãoconcluintes). As variáveis Acesso ao Ambiente do Curso (1 - 0 a 10, 2 - 11 a 20, 3 - acima de 20) e Nível de Instrução $\left(1-\right.$ até $2^{\circ}$ grau, $2-3^{\circ}$ grau incompleto, $3-3^{\circ}$ grau e pós), por terem três categorias, foram transformadas em variáveis dummy (Amb1 e Amb2; Instru1 e Instru2), totalizando dez variáveis antecedentes no modelo testado estatisticamente.

O tratamento escolhido para os casos omissos foi pairwise, já que não houve mais de $5 \%$ de freqüência desses casos nas variáveis analisadas. Não foram encontrados casos extremos, tanto na população quanto nas duas amostras criadas em análises posteriores.

\section{RESULTADOS}

A Tabela 2 apresenta os resultados da análise logística para a população de inscritos. As correlações entre as variáveis, os coeficientes de regressão não padronizados, o intercepto (constante) e o erro padrão, bem como o coeficiente Wald, estão nela disponibilizados. São apresentadas também as estatísticas Cox \& Snell $\mathrm{R}^{2}$, Nagelkerke $\mathrm{R}^{2}$ e a probabilidade dos resultados observados (-2LL).

TABELA 2 - Regressão logística para população.

\begin{tabular}{lllllllllll}
\hline Variáveis & sexo & idade & região & mural & lista & chat & amb1 & amb2 & instru1 & instru2 \\
\hline Idade & $-0,068$ & & & & & & & & & \\
Região & 0,032 & 0,064 & & & & & & & & \\
Mural & 0,015 & $-0,002$ & 0,030 & & & & & & & \\
Lista & 0,024 & 0,001 & 0,078 & $-0,144$ & & & & & & \\
Chat & 0,016 & $-0,068$ & 0,001 & $-0,272$ & $-0,040$ & & & & & \\
amb1 & $-0,003$ & 0,097 & 0,028 & 0,224 & 0,104 & 0,191 & & & & \\
amb2 & $-0,004$ & 0,092 & 0,009 & 0,085 & 0,047 & 0,118 & 0,630 & & & \\
instru1 & $-0,049$ & 0,159 & 0,023 & 0,026 & 0,040 & 0,006 & 0,092 & 0,063 & & \\
instru2 & $-0,082$ & 0,329 & $-0,057$ & 0,026 & 0,007 & 0,015 & 0,042 & 0,018 & 0,429 & \\
\hline B & $-0,065$ & $0,010^{*}$ & 0,090 & $-0,638^{*}$ & $-0,325^{*}$ & $-0,728^{*}$ & $3,176^{*}$ & $1,018^{*}$ & $0,476^{*}$ & $0,201^{*}$ \\
Erro & 0,042 & 0,002 & 0,040 & 0,044 & 0,041 & 0,047 & 0,057 & 0,051 & 0,052 & 0,048 \\
Padrão & & & & & & & & & & \\
WALD & 2,41 & 19,94 & 5,11 & 211,33 & 63,66 & 244,06 & 3052,23 & 393,16 & 84,31 & 17,57 \\
Proporção & 0,94 & 1,01 & 1,01 & 0,53 & 0,72 & 0,48 & 23,95 & 2,77 & 1,61 & 1,22 \\
\hline
\end{tabular}


Constante $=0,153 ; * \mathrm{p}<0,01$

$-2 \mathrm{LL}=16.696,4$

Cox \& Snell $\mathrm{R}^{2}=0,416 \quad$ Nagelkerke $\mathrm{R}^{2}=0,556$

O teste do modelo completo com as dez variáveis antecedentes apresentou uma probabilidade dos resultados observados (-2LL) igual a 16.696,4. Para se avaliar o poder preditivo das variáveis antecedentes, analisam-se as estatísticas Cox \& Snell $\mathrm{R}^{2}$ e Nagelkerke $\mathrm{R}^{2}$, as quais são semelhantes ao $\mathrm{R}^{2}$ do modelo de regressão múltipla linear, mais comumente utilizado em pesquisas. Portanto, de acordo com a Tabela 2, pode-se dizer que 55,6\% da variação no resultado (VD) é explicada pelo modelo de regressão logística. Isso significa idealmente que, em mais da metade dos casos, a evasão (VD) é explicada pelo modelo testado neste estudo.

O teste do modelo completo, comparado com o modelo da constante, apresentou diferença significativa $\left.\chi_{(10, N=19.849)}^{2}=10.660,2, p<0,001\right)$, indicando que oito das 10 variáveis antecedentes diferem significativamente entre concluintes e não concluintes $(\mathrm{p}<0,01)$. Apenas as variáveis sexo e região geográfica não apresentam diferença significativa, apesar de esta última apresentar diferença a $5 \%$ de significância. Em outras palavras, ao analisar a população de inscritos, esta pesquisa indica que alunos que concluem o curso via Internet têm características demográficas (idade) e padrões de acesso ao ambiente eletrônico do curso (acesso ao ambiente, ao chat, à lista de discussão e ao mural de notícias) bastante diferentes daqueles alunos que se evadem.

Outra maneira de avaliar o ajuste do modelo consiste na comparação entre os valores preditos e os resultados realmente observados. O ajuste do modelo foi expressivo, com $87,8 \%$ de concluintes e $75,1 \%$ de não concluintes classificados corretamente. No total, o modelo classificou corretamente $82 \%$ de participantes.

Com o intuito de verificar a ocorrência do erro tipo I, em função do tamanho da amostra, as mesmas análises foram realizadas com duas amostras aleatórias simples, de aproximadamente $7 \%$ dos casos válidos (amostra 1: $\mathrm{n}=1.349$; amostra 2: $\mathrm{n}=1.436$ ). A escolha por fazer a análise em amostras aleatórias e o critério utilizado para delimitação da amostra segue a colocação de Tabachnick e Fidell (2001). A Tabela 3 apresenta os resultados das análises realizadas com a Amostra 1.

\section{Tabela 3 - Regressão logística para amostra 1.}

\begin{tabular}{|c|c|c|c|c|c|c|c|c|c|c|}
\hline Variáveis & sexo & idade & região & mural & lista & chat & $\mathrm{amb} 1$ & $\mathrm{amb} 2$ & instru1 & instru2 \\
\hline idade & $-0,082$ & & & & & & & & & \\
\hline região & 0,002 & 0,009 & & & & & & & & \\
\hline mural & $-0,036$ & 0,058 & 0,032 & & & & & & & \\
\hline
\end{tabular}


EVASÃO EM CURSO VIA INTERNET: EXPLORANDO VARIÁVEIS EXPLICATIVAS

Gardênia da Silva Abbad - Renata Silveira Carvalho - Thaís Zerbini

\begin{tabular}{lllllllllll} 
lista & 0,021 & $-0,015$ & 0,062 & $-0,138$ & & & & & & \\
chat & 0,042 & $-0,092$ & 0,058 & $-0,248$ & $-0,012$ & & & & & \\
amb1 & $-0,010$ & 0,143 & 0,020 & 0,216 & 0,167 & 0,148 & & & & \\
amb2 & 0,007 & 0,139 & 0,019 & 0,092 & 0,073 & 0,091 & 0,645 & & & \\
instru1 & $-0,034$ & 0,149 & 0,019 & 0,063 & $-0,001$ & 0,007 & 0,089 & 0,098 & \\
instru2 & $-0,062$ & 0,354 & $-0,065$ & 0,075 & $-0,016$ & $-0,006$ & 0,019 & 0,055 & 0,438 & \\
\hline B & 0,109 & 0,013 & $-0,012$ & $-0,718^{*}$ & $-0,274$ & $-0,864^{*}$ & $3,081^{*}$ & $1,026^{*}$ & 0,279 & $-0,045$ \\
Erro & 0,158 & 0,009 & 0,152 & 0,166 & 0,156 & 0,179 & 0,219 & 0,200 & 0,193 & 0,187 \\
Padrão & & & & & & & & & & \\
WALD & 0,473 & 2,152 & 0,006 & 18,794 & 3,064 & 23,288 & 198,575 & 26,226 & 2,102 & 0,057 \\
Proporção & 1,11 & 1,01 & 0,99 & 0,49 & 0,76 & 0,42 & 21,77 & 2,79 & 1,32 & 0,96 \\
\hline Constante $=0,274 ; * \mathrm{p}<0,01$ & & & & & & & & & \\
-2LL = 1.145,9 & & & & & & & & & \\
Cox \& Snell R $\mathrm{R}^{2}=0,413$ & Nagelkerke $\mathrm{R}^{2}=0,552$ & & & & & & \\
\hline
\end{tabular}

Os resultados foram distintos da primeira análise no que diz respeito às variáveis Idade, Acesso à Lista de Discussão e Nível de Instrução que, na primeira análise, tiveram contribuição significativa na explicação da evasão, na segunda análise com a amostra 1, não mais contribuíram para a explicação da variável critério. Os resultados indicam, provavelmente, a ocorrência do erro tipo I nas análises com a população, devido ao grande número de casos válidos, o que gerou correlações significativas espúrias com a variável critério. Os resultados das análises com a população, então, devem ser interpretados com cautela, pois podem não ser tão confiáveis quanto os resultados obtidos com a análise das amostras aleatórias.

Os resultados das análises realizadas com a amostra 2 são muito semelhantes aos da amostra 1 e, portanto, não são apresentados aqui. O modelo empírico de explicação de evasão, elaborado a partir dos resultados das análises estatísticas, está representado na Figura 2.

\section{Figura 2 - Modelo empírico de explicação de Evasão.}

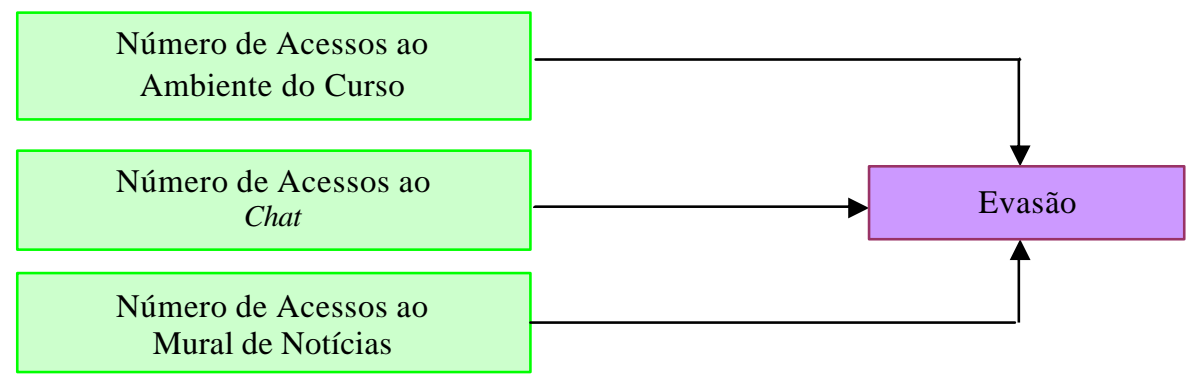

Os resultados indicam que os participantes com poucos acessos aos chats, ao mural de notícias e ao ambiente eletrônico do curso foram aqueles que também tenderam a abandonar o curso. Esses dados sugerem que os evadidos, no período de realização do curso, provavelmente ainda não 


\section{EVASÃO EM CURSO VIA INTERNET: EXPLORANDO VARIÁVEIS EXPLICATIVAS \\ Gardênia da Silva Abbad - Renata Silveira Carvalho - Thaís Zerbini}

dominavam o uso dos recursos baseados nas NTICs e ou não se sentiram estimulados a utilizá-los. Na próxima seção, são apresentadas algumas articulações teóricas entre os resultados aqui obtidos e os estudos anteriores sobre evasão.

\section{CONCLUSÕES E SUGESTÕES}

O modelo de avaliação da evasão em curso a distância, proposto neste estudo, recebeu apoio parcial dos dados empíricos de estudos anteriores. Mostra-se promissora a investigação sistemática da evasão, utilizando-se dados de arquivos secundários e informações sobre a interação da clientela com a interface gráfica do curso. Neste estudo, as variáveis relativas à interação do participante com os recursos eletrônicos do curso mostraram-se importantes variáveis explicativas do perfil que diferencia concluintes de não concluintes de um curso mediado pela Internet.

A baixa freqüência de interação social dos não concluintes com tutores e colegas pode ter sido um dos fatores que levaram aos resultados encontrados nesta pesquisa. Shin e Kim (1999), testando uma análise de regressão logística para investigar os fatores associados à evasão em cursos, descobriram que o uso de atividades face a face associava-se à retenção de alunos, sugerindo que atividades presenciais de interação entre os participantes e os tutores são fatores que diminuem a evasão. Mais estudos são necessários para verificar o quanto a formação de comunidades virtuais de aprendizagem substitui as interações face a face (tutor-aluno e aluno-aluno) e contribui na explicação da persistência em cursos a distância mediados pela Web.

A partir dos resultados encontrados neste estudo, conclui-se que o uso de ferramentas eletrônicas de interação exerce um papel muito importante na retenção do aluno no treinamento. Portanto, sugere-se o desenvolvimento de estratégias que incentivem o participante a utilizar intensivamente os recursos eletrônicos de interação oferecidos durante o curso para que não ocorra a evasão.

É importante ressaltar que o curso avaliado nesta pesquisa tem particularidades que podem ter influenciado os resultados encontrados. Os participantes, por exemplo, não mantinham vínculo empregatíc io com a organização, não estando, por esse motivo, expostos às condições e contingências estabelecidas pela organização enquanto empregadora. Tampouco tiveram que investir financeiramente para inscrever-se no curso, que era totalmente gratuito. Esses fatores, provavelmente, explicam, em parte, o alto percentual de evasão observado neste estudo. 


\section{EVASÃO EM CURSO VIA INTERNET: EXPLORANDO VARIÁVEIS EXPLICATIVAS \\ Gardênia da Silva Abbad - Renata Silveira Carvalho - Thaís Zerbini}

Além disso, o fato de o participante ter tido acesso gratuito ao material impresso pode ter sido responsável pela baixa freqüência de interação espontânea do participante com os recursos eletrônicos do curso. Hábitos arraigados de estudo, provavelmente, prevaleceram e a leitura do material impresso predominou como estratégia de aprendizagem dos participantes. O acesso ao material impresso, entretanto, pode ter sido o motivo principal que levou parte dos não concluintes a se inscreverem no curso. Mais estudos são necessários para investigar as interações entre o uso de materiais impressos e mídias eletrônicas propiciadas pela Web e pela Internet e os níveis de evasão e rendimento dos participantes de cursos multimídias.

Sugere-se a replicação do estudo em outros contextos organizacionais, cursos e amostras, de modo a testar a validade externa e a generalidade desses achados. As organizações que implantaram universidades corporativas deveriam usufruir das vantagens das mídias eletrônicas e analisar os dados armazenados em seus gerenciadores de cursos virtuais, a fim de avaliar seus cursos e aprimorá-los.

Os dados desta pesquisa diferem em parte dos encontrados por Xenos et al. (2002) e Warr e Bunce (1995), uma vez que, no contexto da presente pesquisa, não houve correlações estatisticamente significativas entre idade e evasão. Os mais idosos, na pesquisa de Xenos et al., tendiam a se evadir mais do que os mais jovens. Mais estudos são necessários para se esclarecer essa diferença, mas é provável que ela se deva, em parte, às grandes diferenças existentes entre os contextos das duas pesquisas, em termos de tipo de organização que disponibilizou os cursos estudados, amostras, tipos de cursos, mídias utilizadas e tipo de tutoria, entre outros.

Para aumentar o poder explicativo do modelo de avaliação da evasão, sugerido e testado neste estudo, será necessário acrescentar variáveis relativas ao suporte ambiental à aprendizagem, tal como oferecido por organizações empregadoras, colegas, chefes e familiares. Vários estudos realizados em ambientes não corporativos de aprendizagem aberta mostraram a relevância de variáveis psicossociais (de apoio ou restrição) na explicação de evasão, resultados de aprendizagem e impacto do treinamento no trabalho. Esse é caso dos trabalhos de Carvalho e Abbad (2006), Shin e Kim (1999), Xenos et al. (2002) e Zerbini e Abbad (2005).

Em cursos abertos e oferecidos a distância, as variáveis de suporte devem ser bem definidas. Nesses contextos, por se tratar de cursos disponíveis ao público externo, sem vínculo empregatício com a organização, os fatores situacionais de apoio são relevantes. Entre eles destaque-se a comunidade do aluno, sua família e seu ambiente de trabalho, além de outras variáveis do ambiente macro, como leis, normas, regulamentos e situação política, econômica e social do país. Os fatores do ambiente podem facilitar ou restringir a aprendizagem em cursos presenciais e a distância. No caso desses últimos, o 


\section{EVASÃO EM CURSO VIA INTERNET: EXPLORANDO VARIÁVEIS EXPLICATIVAS \\ Gardênia da Silva Abbad - Renata Silveira Carvalho - Thaís Zerbini}

suporte social de familiares, colegas e amigos pode influenciar a decisão do participante de permanecer no curso até a sua conclusão, como também a sua motivação, aprendizagem e transferência de treinamento para os diferentes contextos e esferas de vida do participante.

O desenho de cursos a distância favorece o estudo em diferentes locais, situações e horários. Essa flexibilidade, entretanto, pode trazer benefícios e novos desafios para o participante, que terá de esforçar-se para conciliar as atividades familiares, profissionais e sociais às necessidades de estudar. Além disso, a clientela de cursos a distância é geralmente formada por adultos que trabalham e dispõem de pouco tempo para os estudos, tal como mostraram os estudos de Shin e Kim (1999) e Xenos et al. (2002).

Futuras pesquisas deveriam, adicionalmente, incluir características motivacionais (valor instrumental do curso para o participante) - como fizeram Lacerda e Abbad (2003) na avaliação de cursos presenciais - e cognitivas (estratégias, estilos e hábitos de estudo) da clientela - a exemplo de Zerbini e Abbad (2005) - em modelos de: diagnóstico de necessidades de treinamento, de avaliação de treinamento e, em especial, em modelos de investigação de evasão em cursos a distância mediados pelas NTICs.

\section{REFERÊNCIAS}

ABBAD, G. Um modelo integrado de avaliação de Impacto do Treinamento no Trabalho - IMPACT. Tese de Doutorado em Psicologia Social e do Trabalho. Brasília: UNB, 1999.

ABBAD, G. et al. Projeto instrucional, aprendizagem, satisfação com o treinamento e auto-avaliação de impacto do treinamento no trabalho. Psicologia: Organizações e Trabalho, v. 1, n. 2, p. 129-161, 2001.

ABBAD, G.; PILATI, R.; PANTOJA, M. J. Avaliação de treinamento: análise da literatura e agenda de pesquisa. Revista de Administração, v. 38, n. 3, p. 181-191, 2003.

ALPERSTEDT, C.; PLONSKI, G. A. As universidades corporativas e sua inserção no setor de educação superior. In: ENCONTRO NACIONAL DA ASSOCIAÇÃO NACIONAL DOS 


\section{EVASÃO EM CURSO VIA INTERNET: EXPLORANDO VARIÁVEIS EXPLICATIVAS \\ Gardênia da Silva Abbad - Renata Silveira Carvalho - Thaís Zerbini}

PROGRAMAS DE PÓS-GRADUAÇÃO E PESQUISA EM ADMINISTRAÇÃO, 25., 2001, Campinas. Anais. Campinas: ANPAD, 2001.

BERNARTH, U.; RUBIN, E. Student retention issues in the online Master of Distance Education (MDE) - an evidence-based approach. In: EDEN Research Workshop, 3., 2004, Oldenburg. Proceedings. Oldenburg: Bibliotheks-und Informationssystem der Universitat, 2004. p. 363-369.

BLOOM, B. et al. Taxonomia dos Objetivos Educacionais - Domínio Afetivo. Porto Alegre: Editora Globo, 1972a.

BLOOM, B. et al. Taxonomia dos Objetivos Educacionais - Domínio Cognitivo. Porto Alegre: Editora Globo, $1972 b$.

BORGES-ANDRADE, J. E. Desenvolvimento de Medidas em Avaliação de Treinamento. Estudos de Psicologia, v. 7, n. especial, p. 31-43, 2002.

CARVALHO, R. S.; ABB AD, G. Avaliação de treinamento a distância: reação, suporte à transferência e impactos no trabalho. Revista de Administração Contemporânea, v. 10, n. 1, p. 96-115, 2006.

CAUBle, A. E.; THURSTON, L. P. Effects of interactive multimedia training on knowledge, attitudes, and self-efficacy of social work students. Research on Social Work Practice, v. 10, n. 4, p. 428-437, 2000.

CHEUNG, D. Developing a student evaluation instrument for distance teaching. Distance Education, v. 19, n. 1, p. 23-34, 1998.

CHEUNG, D. Evidence of a single second-order factor in student ratings of teaching effectiveness. Structural Equation Modeling, v. 7, n. 3, p. 442-460, 2000.

COLQUITT, J. A.; LEPINE, J. A.; NOE, R. N. Toward an integrative theory of training motivation: a meta-analytic path analysis of 20 ye ars of research. Journal of Applied Psychology, v. 85, n. 5, p. 678$707,2000$. 


\section{EVASÃO EM CURSO VIA INTERNET: EXPLORANDO VARIÁVEIS EXPLICATIVAS \\ Gardênia da Silva Abbad - Renata Silveira Carvalho - Thaís Zerbini}

DEAN, A.; WEBSTER, L. Simulations in distance education-progress towards an evaluation instrument. Distance Education, v. 21, n. 2, p. 344-360, 2000.

EBOLI, M. Panorama Brasil. Treinamento e Desenvolvimento, n. 130, ed. Especial, p.22-31, 2003.

FREITAS, I. A.; BORGES-ANDRADE, J. E. Efeitos de Treinamento nos Desempenhos Individual e Organizacional Revista de Administração de Empresas, v. 44, n. 3, p. 44-56, 2004.

GAGNÉ, R. M. The conditions of learning and theory of instruction. New York: Rinehart and Winston, 1985.

GAGNÉ, R. M. Essentials of learning for instruction. Englewood Cliffs, NJ: Prentice Hall, 1988.

HALL, R. H.; WATKINS, S. E.; ELLER, V. E. A model of web based design for learning. In: MOORE, M.; ANDERSON, B. The handbook of distance education. Mahwah, NJ: Erlbaum, 2003. p. 367-376.

HAMBLIN, A. C. Avaliação e controle de treinamento. São Paulo: McGraw-Hill do Brasil, 1978.

LACERDA, E. R. M.; ABBAD, G. Impacto do treinamento no trabalho: investigando variáveis motivacionais e organizacionais como suas preditoras. Revista de Administração Contemporânea, v. 7, n. 4, p. 77-96, 2003.

MAIA, M. C.; MEIRELES, F. S. Tecnologia da informação aplicada na educação a distânc ia no Brasil. In: ENCONTRO NACIONAL DA ASSOCIAÇÃO DOS PROGRAMAS DE PÓS-GRADUAÇÃO E PESQUISA EM ADMINISTRAÇÃO, 27., Atibaia. Anais. Atibaia: ANPAD, 2003.

MOURÃO, L.; BORGES-ANDRADE, J. E. Avaliação de programas públicos de treinamento: um estudo sobre o impacto no trabalho e na geração de emprego. Organizações e Sociedade (no prelo). 


\section{EVASÃO EM CURSO VIA INTERNET: EXPLORANDO VARIÁVEIS EXPLICATIVAS \\ Gardênia da Silva Abbad - Renata Silveira Carvalho - Thaís Zerbini}

PANTOJA, M. J. B.; LIMA, S. M. V.; BORGES-ANDRADE, J. E. Avaliação de impacto de treinamento na área de reabilitação: preditores individuais e situacionais. Revista de Administração, v. 36, n. 2, p. 46-56, 2001.

PORRAS-HERNANDEZ, L. H. Student variables in the evaluation of mediated learning environments. Distance Education, v. 21, n. 2, p. 385-395, 2000.

Roullier, J. Z.; Goldstein, I. L. The relationship between organizational transfer climate and positive transfer of training. Human Resource Development Quarterly, v. 4, n. 4, p. 377-390, 1993.

SALAS, E.; CANNON-BOWERS, J. The science of training: a decade of progress. Annual Review of Psychology, v. 52, n. 1, p. 471-499, 2001.

SALlORENZO, L. H. Avaliação de Impacto do Treinamento no Trabalho: analisando e comparando modelos de predição. Dissertação (Mestrado em Psicologia Social e do Trabalho). Brasília, Universidade de Brasília, 2000.

SCAPIN, D. L.; BASTIEN, J. M. C. Critères ergonomiques pour l'évaluation d'interfaces utilisateurs. Rapports Techniques INRIA-Rocquencourt, v. 156, 1993.

SHEA, T.; MOTIWALLA, L.; LEWIS, D. Internet-based distance education - the administrator's perspective. Journal of Education for Business, v. 77, n. 6, p. 113-117, 2001.

SHIN, N.; KIM, J. An exploratory of learner progress and dropout in Korea National Open University. Distance Education, v. 20, n. 3, p. 81-95, 1999.

TABACHNICK, B. G.; FIDELL, L. S. Using multivariate statistics. New York: HarperCollins, 2001.

VARGAS, M. R. M. Treinamento à distância por videoconferência: o caso da EMBRAPA. In: ENCONTRO NACIONAL DA ASSOCIAÇÃO NACIONAL DOS PROGRAMAS DE PÓSGRADUAÇÃO E PESQUISA EM ADMINISTRAÇÃO, 24., 2000, Florianópolis. Anais. Florianópolis: ANPAD, 2000. 
VEIGA, R. T. et al. O ensino a distância pela Internet: conceito e proposta de avaliação. In: ENCONTRO NACIONAL DA ASSOCIAÇÃO NACIONAL DOS PROGRAMAS DE PÓSGRADUAÇÃO E PESQUISA EM ADMINISTRAÇÃO, 22., Foz do Iguaçu, 1998. Anais. Foz do Iguaçu: ANPAD, 1998.

WARR, P.; BUNCE, D. Trainee characteristics and outcomes of open learning. Personnel Psychology, v. 48 , n. 2 , p. $347-375,1995$.

XENOS, M.; PIERRAKEAS, C.; PINTELAS, P. A survey on student dropout rates and dropout causes concerning the students in the Course of Informatics of the Hellenic Open University. Computers \& Education, v. 39, n. 4, p. 361-377, 2002.

ZERBINI, T.; ABBAD, G. Impacto de treinamento no trabalho via Internet. RAE-eletrônica, v. 4, n. 2 , art. 16, 2005.

\section{Artigo recebido em 13.12.2004. Aprovado em 10.04.2006.}

\section{Gardênia da Silva Abbad}

Professora Adjunta do Departamento de Psicologia Social e do Trabalho, Instituto de Psicologia UnB. Doutora em Psicologia Social e do Trabalho pela UnB.

Interesses de pesquisa nas áreas de aprendizagem em organizações, treinamento, desenvolvimento e educação corporativa, cursos presenciais e a distância e qualificação profissional.

E-mail: gardênia.abbad@gmail.com

Endereço: SQN 205, bloco C, apto. 201, Asa Norte - Brasília - DF, 70843-030.

\section{Renata Silveira Carvalho}

Professora na área de Gestão de Pessoas do Centro Universitário Planalto do Distrito Federal (Uniplan). Consultora especializada em implementações e avaliação de sistemas de programas de treinamento, desenvolvimento e educação corporativa. Mestre em Psicologia Social e do Trabalho pela UnB. 


\section{EVASÃO EM CURSO VIA INTERNET: EXPLORANDO VARIÁVEIS EXPLICATIVAS \\ Gardênia da Silva Abbad - Renata Silveira Carvalho - Thaís Zerbini}

Interesses de pesquisa nas áreas de treinamento, desenvolvimento e educação, presencial e a distância, e de gestão do desempenho humano em organizações e trabalho.

E-mail: renata@ carvalho-net.com

Endereço: SQSW 304, bloco G, apto. 302, Sudoeste - Brasília - DF, 70673-407.

\section{Thaís Zerbini}

Professora na área de Gestão de Pessoas da Pós-Graduação da Universidade Católica de Brasília. Consultora especializada em implementações e avaliação de sistemas de programas de treinamento e desenvolvimento profissional presenciais e a distância. Mestre e doutoranda em Psicologia Social e do Trabalho na UnB.

Interesses de pesquisa nas áreas de treinamento, desenvolvimento e educação - presencial e a distânc ia -, eficácia de ações de educação corporativa e educação profissional - presenciais e a distância.

E-mail: thais.zerbini@gmail.com

Endereço: QRSW 02, bloco B13, apto. 104, Sudoeste - Brasília - DF, 70675-233. 